\title{
PREVALÊNCIA DE PARASITOSES INTESTINAIS ENTRE OS USUÁRIOS DO CENTRO DE SAÚDE DO DISTRITO DE SOUSAS, CAMPINAS, SP (1986-1990)
}

\author{
Ismael Gioia
}

\begin{abstract}
A prevalência das parasitoses intestinais foi levantada nos usuários do Centro de Saúde do Distrito de Sousas; Campinas, SP entre 1986 e 1990. Dentre 770 prontuários observados constatou-se 114 casos positivos (14,8\%) para protozoários, helmintos ou comensais. Ascaris lumbricoides $(48,2 \%)$ seguido de Giardia lamblia $(30,7 \%)$, Trichuris trichiura $(18,4 \%) e$ Enterobius vermicularis $(9,6 \%)$ foram mais prevalentes na faixa etária dos pré-escolares. Os adultos, em maior número na amostra, apresentam-se pouco parasitados. Os demais parasitos e comensais, concorrem com prevalência proporcional aos inquéritos tradicionais realizados na população brasileira, à exceção da ausência de tenídeos e baixa prevalência de Ancylostomatidae. Sugere-se a realização de exame protoparasitológico de rotina entre os préescolares e a utilização de dados dos postos de atendimento primário nos inquéritos parasitológicos.
\end{abstract}

Palavras-chaves: Parasitas intestinais. Helmintos. Prevalência. Inquérito parasitológico.

As infecções parasitárias intestinais continuam endêmicas e devem merecer atenção da saúde pública nos países tropicais. A prevalência geralmente é alta e seu efeito é deletério, principalmente no estado nutricional dos indivíduos infectados 22 .

No Brasil, os levantamentos e inquéritos parasitológicos são feitos a partir da análise do resultado de exames de fezes realizados em laboratórios clínicos ${ }^{41213}$. Por outro lado, vários autores preferem realizar suas pesquisas em comunidades particularmente investigadas para esta finalidade 5151618 .

Em 1968, em cerca de 2,5 milhões de exames coprológicos ${ }^{4}$, encontrou-se uma prevalência de $63 \%$ para Ascaris lumbricoides, $39 \%$ para Trichuris trichiura $28 \%$ para os ancilostomídeos e 2,4\% para Strongyloides stercoralis. Estas prevalências confirmam estatísticas anteriores ${ }^{7}$ e são corroboradas por pesquisas posteriores ${ }^{1}$, tanto para protozooses quanto para helmintíases, demonstrando dificuldades em seu controle.

No Estado de São Paulo ${ }^{6}$ houve uma diminuição gradativa destes índices, entre $1960 \mathrm{e}$ 1979, graças a uma discreta educação e política de

\footnotetext{
Enderego para correspondência: Prof. Ismael Gioja. Depto. de Zoologia, Instituto de Biologia, Universidade Estadual de Campinas. Caixa Postal 6109, 13081-970 Campinas, SP.

Recebido para publicação em $25 / 11 / 91$.
}

melhoria sanitária, além da crescente urbanização, verificadas no Estado. Assim a ascaridíase representa prevalência média de $28,8 \%$, a tricuríase de $22,2 \%$ e a ancilostomíase $20,9 \%$. Giardíase com $10 \%$ e amebíase com $0,8 \%$ completam o quadro das morbidades mais freqüentes.

O Distrito de Sousas, na época do último recenseamento, possuia 7698 habitantes $^{10}$. A maioria de sua população habita zona urbana e o atendimento médico municipal é freqüentado, geralmente, por pessoas das classes sociais menos favorecidas. É comum o atendimento de pessoas que não pertençam a sua área administrativa e não se tem notícia de levantamento parasitológico realizado no distrito.

Os dados aqui apresentados baseiam-se nos casos de parasitoses intestinais verificados na demanda de atendimento primário de uma comunidade. Eles visam quantificar a representatividade desta morbidade através da prevalência de helmintos e protozoários entre os usuários do Centro de Saúde de Sousas.

\section{MATERIAL E MÉTODO}

Os dados foram obtidos nos arquivos do Centro de Saúde Dr. Antonio Pedro Pierro localizado no Distrito de Sousas, Município de Campinas, Estado de São Paulo. Foram analisados 770 prontuários individuais recolhidos de 190 fichas familiares, 
Gioia I. Prevalência de parasitoses intestinais entre os usuários do Centro de Saúde do Distrito de Sousas, Campinas, SP (1986-1990). Revista da Sociedade Brasileira de Medicina Tropical 25:177-182, jul-set, 1992.

abertas entre agosto de 1986 (inauguração da sede atual do serviço) e outubro de 1990 , correspondentes a aproximadamente $5 \%$ das fichas pertencentes ao arquivo de atendimento.

O levantamento foi feito entre setembro e outubro de 1990, desconsiderados os prontuários abertos durante a amostragem, ou em uso no dia da verificação. Sorteada a primeira ficha, foram retiradas esta e uma a cada vinte sucessivas. Destas fichas foram anotados para cada indivíduo o sexo, idade e ausência ou presença de exame parasitológico e seu resultado. Para as pessoas positivas para enterocomensais, protozoários parasitos ehelmintos acrescentou-se idade, época do exame e endereço.

Os indivíduos foram distribuídos nas faixas etárias entre 1 e 7 (pré-escolar), 8 e 18 (adolescente), 19 e 45 (adulto) e 45 ou mais anos (idoso).

Foram tabelados os dados segundo o sexo dos indivíduos amostrados, a freqüência dos parasitos encontrados e sua prevalência nas faixas etárias consideradas.

\section{RESULTADOS}

Dos 770 prontuários individuais examinados, 318 eram do sexo masculino (41,3\%) e 452 do sexo feminino $(58,7 \%)$. Dentre aqueles para quem se solicitou exame parasitológico, $114(14,8 \%)$ apresentaram-se positivos para, pelo menos um, helminto, protozoário ou comensal. Destes, 57 $(50,0 \%)$ eram do sexo masculino e $57(50,0 \%)$ do feminino. A faixa etária que apresentou o maior número na amostragem foi a dos adultos (295 indivíduos) com $24(8,1 \%)$ parasitados. Os préescolares (150 indivíduos) foram os mais intensamente parasitados: $56(37,3 \%)$ casos positivos. Adolescentes, representados na amostra por 201 indivíduos e idosos, 124 indivíduos, apresentaram, respectivamente, $29(14,4 \%)$ e 5 $(4,0 \%)$ de parasitismo.

Para um total de 144 exames parasitológicos solicitados, 30 indivíduos apresentaram resultado negativo. Somente 7 pessoas $(6,1 \%)$ dentre os parasitados apresentaram retorno ao serviço com exame negativo (após tratamento).

O uniparasitismo ocorreu em $71(62,2 \%)$ indivíduos e destes, $13(11,4 \%)$ apresentaram-se com exames que reveleram somente a presença de comensais. O poliparasitismo também foi comum:
43 pessoas albergavam mais que um parasito. $\mathrm{O}$ exame de fezes revelou que 33 pessoas abrigavam duas espécies, 7 três, 2 quatro e 1 indivíduo sete espécies diferentes.

A associação mais freqüente $(12,2 \%)$ ocorreu entre os patogênicos Ascaris lumbricoides (Linnaeus, 1758) e Trichuris trichiura (Linnaeus, 1771) Stiles, 1901 (14 casos). A. lumbricoides e Giardia lamblia Stiles, 1915 contribuíram com 10 casos e a associação entre os 3 parasitos foi verificada em 4 pessoas.

Destaca-se a prevalência (Tabela 1), dentre os protozoários parasitos de $G$. lamblia $(30,7 \%)$, mais freqüente nos pré-escolares, com relativo equilíbrio entre os sexos, e com decréscimo substancial a partir dos 8 anos (Tabela 2). O parasito mais expressivo (Tabela 1) foi o helminto $A$. lumbricoides $(48,2 \%)$ com equilíbrio entre os sexos e também apresentando decréscimo de prevalência a partir dos 8 anos (Tabela 2). Vem a seguir $T$. trichiura $(18,4 \%)$ com predominio parasitário sobre as mulheres (Tabela 1) e prevalência $(8,0 \%)$ nitidamente maior na faixa entre 1 e 7 anos (Tabela 2). Com prevalência de $9,6 \%$ encontramos Enterobius vermicularis (Linnaeus, 1758) Leach, 1853 (Tabela 1). Este helminto é freqüente entre os pré-escolares e ausente nos adultos e idosos (Tabela 2). Os ancilostomídeos foram diagnosticados especialmente nas mulheres em todas as faixas etárias (Tabela 2) com prevalência acentuada $(2,3 \%)$ entre os adultos. Hymenolepis nana (Siebold, 1852) Blanchard, 1891 e Strongyloides stercoralis (Bavay, 1876) Stiles \& Hassal, 1902 (diagnosticado em 3 mulheres) concorrem respectivamente com prevalência de $3,5 \%$ e $2,6 \%$. A mesma também é baixa para Entamoeba histolytica Schaudinn, 1903: $1,7 \%$. Os adultos e idosos não apresentaram os 3 últimos parasitos. O helminto Schistosoma mansoni Sambon, 1907 foi detectado em 3 indivíduos (sendo duas mulheres) que na amostra significa prevalência de $0,3 \%$ e entre os positivos de $2,6 \%$. Somente 18 pessoas habitavam a zona rural e destas 3 apresentaram comensais. As 15 restantes portavam A. lumbricoides $(46,6 \%)$, G. lamblia $(20,0 \%), T$. trichiura, S. stercoralis, E. vermicularis e Ancilostomídeos (13,3\%).

O flagelado Chilomastix mesnili (Wenyon, 1910) Alexeieff, 1912 foi diagnosticado nas fezes de uma única pessoa, o mesmo acontecendo com lodamoeba bütschlii (Prowazek, 1911) Dobell, 1919. 
Gioia I. Prevalência de parasitoses intestinais entre os usuários do Centro de Saúde do Distrito de Sousas, Campinas, SP (1986-1990). Revista da Sociedade Brasileira de Medicina Tropical 25:177-182, jul-set, 1992.

Tabela 1 - Distribuição de protozoários e helmintos nơs indivíduos amostrados no Centro de Saúde de Sousas Campinas, SP.

\begin{tabular}{|c|c|c|c|c|c|}
\hline \multirow{2}{*}{ Parasitos/comensais } & \multicolumn{3}{|c|}{ Indivíduos parasitados } & \multicolumn{2}{|c|}{ Prevalência (\%) } \\
\hline & masc. & fem. & Total & na amostra ${ }^{(1)}$ & nos parasitados ${ }^{(2)}$ \\
\hline Giardia lamblia (Gl) & 19 & 16 & 35 & 4,5 & 30,7 \\
\hline Endolimax nana (En) & 6 & 7 & 13 & 1,6 & 11,4 \\
\hline Entamoeba coli $(E c)$ & 6 & 4 & 10 & 1,3 & 8,7 \\
\hline Entamoeba histolytica (Eh) & 1 & 1 & 2 & 0,2 & 1,7 \\
\hline lodamoeba bütschlii & 1 & - & 1 & 0,1 & 0,8 \\
\hline Chilomastix mesnili & - & 1 & 1 & 0,1 & 0,8 \\
\hline Ascaris lumbricoides (Al) & 27 & 28 & 55 & 7,4 & 47,0 \\
\hline Trichuris trichiura (Tt) & 7 & 14 & 21 & 2,7 & 18,4 \\
\hline Enterobius vermicularis (Ev) & 7 & 4 & 11 & 1,4 & 9,6 \\
\hline Ancylostomatidae (An) & 2 & 8 & 10 & 1,2 & 8,7 \\
\hline Hymenolepis nana $(\mathrm{Hn})$ & 2 & 2 & 4 & 0,5 & 3,5 \\
\hline Schistosoma mansoni (Sm) & 1 & 2 & 3 & 0,3 & 2,6 \\
\hline Strongyloides stercoralis (Ss) & - & 3 & 3 & 0,3 & 2,6 \\
\hline
\end{tabular}

${ }^{(1)} \mathrm{n}=770 \quad(2) \mathrm{n}=114$

Tabela 2 - Prevalência dos protozoários e helmintos mais frequentes nos indivíduos amostrados segundo a faixa etária

\begin{tabular}{|c|c|c|c|c|c|c|c|c|c|c|c|c|c|}
\hline \multirow{2}{*}{$\begin{array}{l}\text { Faixa } \\
\text { etária } \\
\text { (anos) }\end{array}$} & \multirow[b]{2}{*}{$\mathbf{N}$} & \multirow[b]{2}{*}{ NP } & \multicolumn{11}{|c|}{$\begin{array}{l}\text { Espécies encontradas* } \\
\mathrm{n}^{0} \text { portadores / (prevalência na faixa etária - \%) }\end{array}$} \\
\hline & & & Eh & $E_{\mathcal{C}}$ & En & Gl & A1 & $\mathrm{Tt}$ & $\mathrm{Ev}$ & An & $\mathrm{Hn}$ & $\mathrm{Sm}$ & Ss \\
\hline $1-7$ & 150 & 56 & $1(0,6)$ & $3(2,0)$ & $5(3,3)$ & $25(16,6)$ & $28(18,6)$ & $12(8,0)$ & $9(6,0)$ & $1(0,6)$ & $2(1,3)$ & - & $1(0,6)$ \\
\hline $8-18$ & 201 & 28 & $1(0,4)$ & $3(1,4)$ & $1(0,4)$ & $8(3,9)$ & $16(7,9)$ & $5(2,4)$ & $2(0,9)$ & $1(0,4)$ & $2(0,9)$ & - & $2(0,9)$ \\
\hline $19-45$ & 295 & 25 & - & $4(1,3)$ & $4(1,3)$ & $1(0,3)$ & $11(3,7)$ & $3(1,0)$ & - & $7(2,3)$ & - & $3(1,4)$ & \\
\hline$>45$ & 124 & 5 & - & . & $3(2,4)$ & $1(0,8)$ & - & $1(0,8)$ & - & $1(0,8)$ & - & 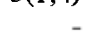 & \\
\hline
\end{tabular}

$\mathrm{N}=\mathrm{n}^{\circ}$ de individuos observados, $\mathrm{NP}=\mathrm{n}^{\circ}$ de indivíduos parasitados

* abreviações da Tabela 1

O perfil parasitológico da amostra se completou com o diagnóstico, através do exame clínico, de cinco casos de larva migrans cutânea, dois casos de berne e um de escabiose.

\section{DISCUSSÃO}

Não há evidências de que a amostra represente o universo de pessoas atendidas no Centro de Saúde de Sousas. A diversidade dos parasitos e comensais encontrados, sua distribuição e proporção no conjunto dos prontuários observados são, entretanto, semelhantes ao verificado no Estado de São Paulo ${ }^{6}$.
Por outro lado, diversos autores distribuem seus resultados em classes de idade não padronizadas ${ }^{11}$ 141920 . O intervalo é arbitrário e quase sempre não é justificado. As faixas etárias aqui adotadas contemplam o pré-escolar, o adolescente, o adulto e o idoso, ainda que esta divisão seja discutível sob o aspecto dos critérios levados em consideração. É plausível, entretanto, admitir que em cada uma destas faixas o indivíduo está socialmente desempenhando atividades que o colocam, sob o ponto de vista da aquisição das parasitoses, frente a riscos diferenciados. Três outros aspectos merecem ser salientados: 1. não é rotina a solicitação de 
Gioia I. Prevalência de parasitoses intestinais entre os usuários do Centro de Saúde do Distrito de Sousas, Campinas, SP (1986-1990). Revista da Sociedade Brasileira de Medicina Tropical 25:177-182, jul-set, 1992.

exame de fezes no atendimento do Centro de Saúde; 2. na maioria das vezes o resultado do exame foi transcrito para o prontuário, dificultando a identificação da metodologia utilizada e avaliar sua eficiência e especificidade para diversos parasitos; 3. o retorno ao serviço de posse de exame negativo, após prescrição de tratamento, parece ser negligenciado pelos pacientes.

A prevalência de $14,8 \%$ de indivíduos parasitados sugere que deve ser dada maior atenção para esta morbidade, pois seu conhecimento é fundamental no desenvolvimento e planejamento da atenção médica.

As parasitoses intestinais têm em comum a disseminação pela contaminação fecal ${ }^{9}$. Apresentam, entretanto, diferenças epidemiológicas. As helmintíases geralmente provém de contaminação fecal do solo e de inadequado saneamento ambiental e as protozooses de deficiente higiene individual, pois geralmente se transmitem de pessoa a pessoa.

Os altos índices verificados para $A$. lumbricoides, T. trichiura e G. lamblia refletem ambas características epidemiológicas presentes em uma população do tipo urbano e nível sanitário baixo $0^{9}$. A idade pré-escolar apresenta a maior intensidade de parasitismo e o helminto mais freqüenteé $A$. lumbricoides, como ocorrena maioria dos inquéritos realizados no Brasil. Já a presença de protozoários comensais demonstra a contaminação de alimentos por material fecal. A baixa incidência de Ancylostomatidae possivelmente deve estar relacionada a baixa sobrevivência de suas formas infectantes em solos não arenosos presentes na região, mais favoráveis ao desenvolvimento dos ovos de $A$. lumbricoides e T. trichiura ${ }^{2}$. H. nana e E. vermicularis são helmintos com infecção mais prevalente na infância com profilaxia ligada a higiene pessoal ${ }^{17}$. Os enterocomensais Entamoeba coli (Grassi, 1879; Casagrandi \& Barbagallo, 1895) e Endolimax nana (Wenyon \& O'Connor, 1917; Brug, 1918) são prevalentes em ambos os sexos e em várias faixas etárias. Sua transmissão provavelmente está ligada ao consumo de vegetais mal lavados. Quanto à presença de $S$. mansoni, (3 casos não autóctones) sabe-se que o Estado de São Paulo tem recebido grande fluxo migratório oriundo de zonas endêmicas. É prioritário o estabelecimento de uma política de saúde ${ }^{6}$ que vise o diagnóstico e tratamento específico destas fontes de infecção.
Notou-se a ausência de cestóides (Taenia solium Linnaeus, 1758 e T. saginata Goeze, 1782) possivelmente pela vocação de pecuária de gado leiteiro e não de corte, além da baixa população de suínos, na região.

Este levantamento repete, no tocante à prevalência, as proporções de qualquer inquérito parasitológico específico já realizado, à exceção de Ancylostomatidae, que aparece com baixa incidência, curiosamente maior nos adultos. Alguns helmintos foram verificados com predomínio entre as mulheres, provavelmente por serem estas atendidas no programa de exame pré-natal do Centro, onde é freqüente a solicitação de exame de fezes.

O exame coprológico poderia ser rotineiro, principalmente nos pré-escolares, pois como salienta Vinha ${ }^{21}$, "a redução das condições físicas e de atividades de cada parasitado multiplicado pelo expressivo contingente de doentes/portadores deve representar uma perda óbvia previsível de incontáveis dias de trabalho, de capacidade para o aprendizado, de atraso no desenvolvimento físico e mental, social e comunitário".

Os resultados verificados atestam a representatividade da amostra obtida ("porta de entrada" do sistema) sugerindo não só a importância de sua utilização no levantamento do quadro parasitológico da comunidade atendida, mas também na avaliação da qualidade parasito-sanitária da região geográfica da unidade primária de saúde.

\section{AGRADECIMENTOS}

Sinceros agradecimentos à médica Anna Thereza B. C. G. Sousa Pinto, do Centro de Saúde de Sousas, à Dra. Maria Lúcia Lebrão (HEP-724) e ao Dr. José Maria Barata (ambos da Fac. de Saúde Pública - USP) e à Dra. Rosa M. D. Souza Dias (Inst. Adolfo Lutz) pelas oportunas sugestões e críticas.

\section{SUMMARY}

The prevalence of intestinal parasites was carried on users of the Health Center of Sousas District, Campinas, SP (1986-1990). From 770 observed medical records we verify 114 positive cases (14.8\%) for protozoa, helminths and comensals. Ascaris lumbricoides (48.2\%), Giardia 
Gioia I. Prevalência de parasitoses intestinais entre os usuários do Centro de Saúde do Distrito de Sousas, Campinas, SP (1986-1990). Revista da Sociedade Brasileira de Medicina Tropical 25:177-182, jul-set, 1992.

lamblia (30.7\%), Trichuris trichiura (18.4\%) and Enterobius vermicularis $(9.6 \%)$ were more prevalent in pre-school children. The adults, what are the most numerous class, appeared few parasitised. The other parasites and comensals appeared with a prevalence like the traditional brazilian population surveys, except for the absence of tenids and low prevalence of
Ancylostomatidae. We propose the realization of routine stool parasitological examinations among the pre-school children and the use of the primary health care data in parasitological survey.

Key-words: Intestinal parasites. Helminths. Occurrence. Parasitological survey.

\section{REFERÊNCIAS BIBLIOGRÁFICAS}

1. Baruzzi RG, Coutinho JO. Geografia médica das heimintíases e geografia médica das protozooses $I n$ : Lacaz CS, Baruzzi RG, Siqueira Jr W (ed) Introdução à Geografia Médica do Brasil. Editora Edgard Blücher Ltda, p.568, 1972.

2. Beaver PC. Observations on the epidemiology of Ascariasis in a region of high hookworm endemicity. Parasitology 38:445-453, 1952.

3. Botero D. Persitencia de parasitoses intestinais endemicas en America Latina. Boletin de la Oficina Sanitaria Panamericana 90:39-47, 1981.

4. Camillo-Coura L. Contribuição ao estudo das geohelmintiases. Tese de Livre Docência, Universidade Federal do Rio de Janeiro, Rio de Janeiro, 1970.

5. Castro C, Molina LA. As parasitoses intestinais na população do município de Mambaí em dois períodos distintos, 1974 e 1986. Brasil Médico 25:5-7, 1988.

6. Chieffi PP, Waldman EA, Waldman CCS, Sakata EE, Gerbi LJ, Rocha AB, Aguiar PR. Aspectos epidemiológicos das enteroparasitoses no Estado de São Paulo, Brasil. Revista Paulista de Medicina 99:34-36, 1982.

7. Coutinho JO, Silvany Filho A. Notas sobre inquérito coprológico efetuado em pacientes internados no Hospital de Santa Isabel, Salvador, Bahia. Anais da Faculdade de Medicina, Universidade de São Paulo 25:56-64, 1950.

8. Evangelista A, Komma MD, Santos MAQ. Prevalência de parasitoses intestinais em Goiânia. Revista de Patologia Tropical 1:51-61, 1972.

9. Gonçalves A, Andrade JCR, Giribola L, Oliveira MC. Levantamento das parasitoses intestinais e condições sócio-econômicas e sanitárias em bairro de Botucatu, SP. Revista da Sociedade Brasileira de Medicina Tropical 7:25-43, 1973.

10. Instituto Brasileiro de Geografia e Estatística. IX Recenseamento Geral do Brasil - 1980, São Paulo, v1 t3, no. 17 p.90, 1982.

11. Kosoff P, Hernandez F, Pardo V, Visconti M, Zimmerman M. Urban helminthiasis in two socioeconomically distinct Costa Rican communities. Revista de Biologia Tropical 37:181-186, 1989.

12. Mello A, Mello NR, Bourroul GP, Gomes LFS Verificações helmintológicas em 3.000 exames de fezes. Revista do Instituto Adolfo Lutz 19:75-85, 1959.

13. Mello NR, Mello A, Bourroul GP, Gomes LFS Protozoários em 3.000 exames de fezes. Revista do Instituto Adolfo Lutz 19:87-95, 1959.

14. Nwosu ABC. The community ecology of soiltransmitted helminth infections of humans in a hyperendemic area of Southern Nigeria. Annals of Tropical Medicine and Parasitology 75:197-203, 1981.

15. Pedrazzani ES, Mello DA, Pripas S, Fucci M, Barbosa CAA, Santos MCM. Helmintoses intestinais. II. Prevalência e correlação com renda, tamanho da família, anemia e estado nutricional. Revista de Saúde Pública 22:384-389, 1988

16. Perez MD, Artigas PT, Lollo N. Levantamento parasitológico realizado na cidade de Bragança Paulista (Estado de São Paulo), com contribuição ao levantamento da carta planorbídica do Estado de São Paulo. Revista da Sociedade Brasileira de Medicina Tropical 7:167-176, 1973.

17. Pessoa SB, Martins AV. Pessoa - Parasitologia Médica. $11^{\mathbf{a} .}$ edição, Editora Guanabara Koogan, 1982.

18. Santos RV, Coimbra Junior CEA, Ott AMT. Estudos epidemiológicos entre grupos indígenas de Rondônia. III. Parasitoses intestinais nas populações dos vales dos rios Guaporé e Mamoré. Cadernos de Saúde Pública 1:467-477, 1985.

19. Schenone H, Rojas A, Galdames M, Villarroel F. Aspectos epidemiologicos de las infecciones humanas 
Gioia I. Prevalência de parasitoses intestinais entre os usuários do Centro de Saúde do Distrito de Sousas, Campinas, SP (1986-1990). Revista da Sociedade Brasileira de Medicina Tropical 25:177-182, jul-set, 1992.

por protozoos y helmintos intestinales en Chile (1970-1980). Boletin Chileno de Parasitologia 36:4448, 1981.

20. Sole TD, Croll NA. Intestinal parasites in man in Labrador, Canada. The American Journal of Tropical Medicine and Hygiene 29:364-368, 1980.

21. Vinha $C$. Necessidade de uma política sanitária nacional para o combate às parasitoses intestinais. Revista da Sociedade Brasileira de Medicina Tropical 10:297-301, 1975.

22. World Health Organization. WHO Expert Committee on prevention and control of intestinal protozoan and helminthic infections. WHO Technical Report Series $n^{0} \cdot 666,1981$. 